Gerhard Schwediauer - Wiener Börse AG

\section{Die Leitidee der Nachhaltigkeit und ihre Konsequenzen für die Praxis der Forschungsförde- rung zum Globalen Wandel}

\author{
Ein Vorwort von Norbert Binder \\ BMBF, Referat 622 - Globaler Wandel
}

Das BMBF hat seine Fördermaßnahmen auf dem Gebiet der Global Change-Forschung unter die politische Leitidee der Nachhaltigkeit, verstanden als Koevolution von Natur und Anthroposphäre, gestellt. Die in diesem Kontext geförderten Forschungsvorhaben sollen dazu beitragen, eine ökonomisch leistungsfähige, sozial gerechte und ökologisch verträgliche Entwicklung in Gang zu setzen. Menschliches Leben soll stabilisiert und qualifiziert verbessert werden, ohne die Tragfähigkeit der Ökosysteme zu gefährden (siehe hierzu: Grundprinzipien der Biodiversitätsforschung durch das BMBF Empfehlungen eines Sachverständigenausschusses „Biodiversität und Nachhaltigkeit; Vorsitz Prof. U.E. Simonis; Frühjahr 2003).

Ein nur sehr allgemein formuliertes politisches Leitziel für die Forschungsförderung, wie das der Nachhaltigkeit, das das gesamte Spektrum wirtschaftlicher und gesellschaftlicher Entwicklungspotenziale integrieren und auch nutzen möchte, bedarf auf der Ebene der Projektorganisation und -durchführung konkreter Aussagen über die jeweilige Förderstrategie auf zum Teil sehr weit auseinander liegenden Themenbereichen.

Das BMBF hat daher im Kontakt mit Wissenschaftlern und Gremien themenspezifische Überlegungen zur Konkretisierung seiner Nachhaltigkeitsstrategie angeregt. Diese sollen spezielle Förderschwerpunkte, wie in diesem Fall den der Biodiversität, inhaltlich konkretisieren. Die Spezifikationen sollen es gleichzeitig erlauben, die Leitidee der Nachhaltigkeit auch für Zwecke der Ausschreibung von Förderthemen und der Organisation von Forschungsverbünden zu operationalisieren.

Inhaltliche Elemente des auf das generelle Leitthema der Nachhaltigkeit bezogenen Begriffs der Biodiversität sind die Erhaltung der biologischen Vielfalt, die nachhaltige Nutzung ihrer Bestandteile und die gerechte Aufteilung der sich aus der Nutzung biologischer Ressourcen ergebenden Vorteile.

ITAS hat exemplarisch das Thema „Biodiversität und Nachhaltigkeit" mit Blick auf diese genannten Teilziele untersucht und Konsequenzen für Forschung und Forschungsförderung abgeleitet. Das BMBF dankt den Autoren dafür, dass sie sich dieser für die künftige Erörterung von Nachhaltigkeitsstrategien in der Fachwelt wie auch für die Förderpraxis wichtigen Aufgabe angenommen haben und ihre Thesen nunmehr publik machen. Es hofft auf eine lebhafte Diskussion, deren Ergebnisse ebenfalls Eingang in die etablierten Verfahren der Forschungsförderung finden können.

\section{Das Fallbeispiel „Biodiversität“"1}

von J. Kopfmüller, ITAS

\section{Nachhaltige Entwicklung und Globaler Wandel}

Seit mittlerweile rund zwanzig Jahren existiert der Begriff des Globalen Wandels, verbunden mit umfangreichen Forschungsanstrengungen auf verschiedenen Ebenen. Ähnlich lange befindet sich der Begriff der nachhaltigen Entwicklung in der politischen und gesellschaftlichen Debatte, wird über seine konzeptionelle und operative Ausgestaltung gearbeitet. Die Tatsache, dass bislang eine - prinzipiell wünschenswerte - Verknüpfung dieser beiden Themenfelder auf der Ebene der Forschungsförderung und der Forschungsprojekte noch nicht in hinreichendem Maße gelungen ist, stellt einen wesentlichen Hintergrund und Anlass für die in diesem Beitrag skizzierte Aktivität dar.

Was die Phänomene des globalen Wandels anbelangt, ist zunächst - korrespondierend mit dem im einleitenden Beitrag zu diesem Schwerpunkt von J. Jörissen beschriebenen Nachhaltigkeitsverständnis des integrativen Konzepts und 
den dort formulierten Leitlinien - festzustellen, dass hier zwei Typen zu unterscheiden sind:

- Zum einen solche bezogen auf das Verhältnis Gesellschaft - Natur: Ressourcenverbrauch und die Nutzung der Umwelt als Senke sind Themen, die zu den einschlägigen Problemen globaler Umweltveränderungen führen (z. B. Biodiversitätsrückgang, Wasserqualität und -verfügbarkeit, Bodendegradation oder Klimawandel). Die Frage, wie wir das Verhältnis von Anthroposphäre und natürlicher Umwelt nachhaltig gestalten können, ist weithin ungeklärt. Ihre Beantwortung bedarf sowohl fundierten wissenschaftlichen Wissens als auch gesellschaftlichpolitischer Verständigungsprozesse auf nationaler und internationaler Ebene. Dies betrifft die Frage der Formulierung von Entwicklungszielen ebenso wie die Gestaltung und Umsetzung politischer Handlungsstrategien zur Erreichung dieser Ziele. Dabei sind die Folgen globaler Umweltveränderungen (z. B. Konflikte um Ressourcen, Migration usw.) wie auch die unterschiedlichen Wirkungen von Handlungsstrategien von erheblicher sozialer, ökonomischer und politischer Reichweite.

- Zum anderen existieren solche Phänomene bezogen auf gesellschaftsimmanente Entwicklungen: Eine ganze Reihe globaler Nachhaltigkeitsdefizite bzw. problematischer Phänomene des globalen Wandels bestehen, was ihre Ursachen und Wirkungen anbelangt, unabhängig von ökologischen Aspekten. Neben den Gesellschaft - Natur-Beziehungen müssen deswegen auch die „Gesellschaft - Gesellschaft-Perspektiven" betrachtet werden. So kennzeichnet beispielsweise die ökonomische Globalisierung ein tief greifendes und folgenreiches Element des Globalen Wandels. Unter dem Aspekt nachhaltiger Entwicklung ist hier vor allem die Ausgestaltung der verschiedenen Facetten weltwirtschaftlicher Rahmenbedingungen $\mathrm{zu}$ nennen. Die hier zu diagnostizierenden Benachteiligungen verschiedenster Art insbesondere der ärmsten Staaten werden häufig in gleicher Weise als ein wesentlicher Faktor für das Entstehen bzw. die Verstärkung von Problemen wie Armut, Hunger oder Verschuldung angeführt wie für ökologische Probleme. Daher stellt die Realisierung von
Fairness und Gerechtigkeit in den weltwirtschaftlichen Rahmenbedingungen eine wesentliche Herausforderung für die Umsetzung einer global nachhaltigen Entwicklung dar. Bedeutsam ist dabei, wie Institutionen, Regime und Prozesse auf den verschiedenen Ebenen von (Global) Governance beschaffen sein müssten, um nachhaltige Entwicklung zu ermöglichen oder zu fördern.

Beide Typen von Phänomenen müssen in gleicher Weise Gegenstand einer (globalen) Forschung zum Globalen Wandel sein, die den Anspruch hat, ein ganzheitliches integratives Nachhaltigkeitskonzept angemessen einzubeziehen.

\section{Herausforderungen für die Forschung}

Um den globalen Wandel vor dem Hintergrund des Nachhaltigkeitsleitbilds analysieren und gestalten zu können, kommt der Forschung erhebliche Bedeutung zu. Dabei geht es primär um die Erzeugung dreier Wissenstypen: Zunächst zielt Erklärungswissen auf ein angemessenes Verständnis bezüglich natürlicher und sozialer Systeme sowie der Wechselwirkungen zwischen menschlichem Wirtschaften und der natürlichen Umwelt und ist notwendige Bedingung für nachhaltigkeitsorientiertes Handeln. Handlungswissen ist idealtypisch als umfassendes und vorausschauendes Wissen über Maßnahmen zur Lösung von Nachhaltigkeitsproblemen, deren Wirkungen und unbeabsichtigte Nebenwirkungen zu verstehen und stellt eine entscheidende Voraussetzung für informierte Entscheidungsfindungen dar. Hierzu gehört auch, die Unsicherheit und Unvollständigkeit des Wissens transparent zu machen und geeignete Wege zum Handeln unter Unsicherheit aufzuzeigen. Schließlich bedarf es des Orientierungswissens, um Kriterien für die Bewertung gesellschaftlicher Zustände und Entwicklungen, globaler Trends und politischer Maßnahmen bereitzustellen, die nachvollziehbare Unterscheidungen zwischen „nachhaltig“ und „nicht bzw. weniger nachhaltig" erlauben. Hierbei sind beispielsweise auch Aspekte der Chancengleichheit und die Umsetzung von Forderungen im Rahmen der Gender-Thematik zu berücksichtigen.

Politisch nutzbare Wissensbestände bestehen in der Regel aus Kombinationen dieser 
Wissenstypen. Ihre Generierung stellt für das Wissenschaftssystem eine neuartige Herausforderung dar. Die klassische Struktur und Entwicklung der Wissenschaften hin zu immer größerer Spezialisierung ist für diese Herausforderungen allein nicht zielführend. Sie muss ergänzt werden um eine neue „Kultur“ integrativer Forschung, die Disziplinengrenzen überschreitet, die international angelegt ist, und die den Bogen von der Grundlagenforschung bis hin zu konkreten Anwendungen spannt.

In Forschungsprojekten zur Nachhaltigkeit im Globalen Wandel können Schwerpunkte in unterschiedlicher Weise auf die genannten Wissenstypen verteilt sein. Wichtig ist jedoch die Realisierung einer integrativen Forschung, in der Verbindungen zwischen allen drei Wissenstypen in dem jeweiligen Themenfeld hergestellt werden. Es bietet sich an, diesen neuen Typ von Forschung durch Modellprojekte zu initiieren, aus denen dann auch für zukünftige Projekte und Programme Lernprozesse entstehen können. Diese Modellprojekte sollten folgende Voraussetzungen erfüllen:

- Sie sollten einen Beitrag zur Verbesserung von Entscheidungsgrundlagen in komplexen Fragen des Globalen Wandels leisten (Handlungsorientierung). Sie müssen sich also mit Fragen der Zustandsanalyse, der Zielformulierung und geeigneter Zielerreichungsstrategien befassen.

- Sie sollten integrativ vor allem in dreierlei Hinsicht sein (vgl. Grunwald 2001): erstens in Bezug auf wissenschaftliche Disziplinen. Bereits die Themenstellung muss interdisziplinär erfolgt sein und Einfluss auf die Arbeitsweise in den beteiligten Disziplinen haben. Deren bloße Addition reicht nicht aus. Insbesondere ist eine Verzahnung von Naturund Sozialwissenschaften erforderlich, wie dies die komplexe und interdependente Natur des Globalen Wandels genauso wie das integrative Nachhaltigkeitskonzept nahe legen. Daneben ist auch eine transdisziplinäre Herangehensweise, d. h. die Einbeziehung auch nicht-wissenschaftlichen Wissens, erforderlich. Integrativität bezieht sich zweitens auf gesellschaftliche Sektoren oder Aktivitätsfelder sowie deren Spezifika und Wechselbeziehungen untereinander. Drittens bezieht sie sich auf die Dimensionen gesellschaftlicher Entwicklung (Ökologie, Ökonomie, Soziales usw.), realisierbar durch eine Orientierung an den angesprochenen, dimensionenübergreifenden Nachhaltigkeitsregeln.

- Weitere, zum Teil für die Umsetzung von Integrativität wichtige Kriterien sind: die Fokussierung auf die drängendsten Probleme innerhalb des Themenbereichs; die Verknüpfung von Grundlagenforschung mit anwendungsbezogener Forschung; die Orientierung auf die Folgen gesellschaftlichen Handelns, auch politischer Maßnahmen, in längerfristiger Perspektive; die Verbindung zwischen lokaler/regionaler und globaler Perspektive sowie die Einbeziehung der wichtigen Akteure mit Blick auf die gesellschaftlichen Umsetzungsmöglichkeiten hinsichtlich Zielformulierungen und Handlungsstrategien (vgl. Hennen, Krings 1998).

- Die Projekte sollten in internationaler Kooperation und eingebunden in internationale Forschungsprogramme durchgeführt werden. Dem WissenschaftlerInnenaustausch und dem capacity-building sind dabei angemessene Bedeutung einzuräumen.

\section{Das Themenfeld Biodiversität}

Gemäß der im Rahmen der Rio-Konferenz 1992 von über 150 Staaten unterzeichneten Biodiversitäts-Konvention wird Biodiversität verstanden als Summe der drei Komponenten: Gesamtheit aller Tier- und Pflanzenarten, genetische Vielfalt innerhalb der Arten sowie Gesamtheit der Ökosysteme. In dieser Konvention wurden - vor dem Hintergrund der Nachhaltigkeitsdebatte - die Grundlagen einer Neuorientierung für den Arten- und Naturschutz festgeschrieben. Artikel 1 beschreibt den zentralen Ziele-Dreiklang aus Erhaltung und nachhaltiger Nutzung der Vielfalt sowie der gerechten Verteilung (national und international) der sich daraus ergebenden Vorteile. In der Forschung zur Biodiversität und zu ihrem innerhalb der letzten Jahrzehnte zu beobachtenden kontinuierlichen Rückgang wird neben der naturwissenschaftlichen zunehmend auch die sozialwissenschaftliche Perspektive eingenommen.

Von entscheidender Bedeutung für den politischen wie auch wissenschaftlichen Umgang mit dem Thema ist die weitere Konkretisierung 
der relativ allgemein formulierten Ziele der Konvention. Anhaltspunkte hierfür kann das HGF-Verbundprojekt „Global zukunftsfähige Entwicklung - Perspektiven für Deutschland“" liefern, in dem das Thema Biodiversität auf verschiedenen Analyseebenen betrachtet wurde.

\section{Ebene der Nachhaltigkeitsregeln}

Zentrale Regel in Bezug auf Biodiversität ist diejenige zur nachhaltigen Nutzung erneuerbarer Ressourcen, zu denen allgemein die Biodiversität gezählt wird (vgl. Kopfmüller et al. 2001). Im Sinne eines erweiterten utilitaristischen Verständnisses begründet sich diese Leitlinie aus drei unterschiedlichen nutzungsabhängigen Wertzuschreibungen (vgl. WBGU 2000): Erstens dem direkten Nutzwert für Produktionsund Konsumzwecke in unterschiedlichen Bereichen. Zweitens dem optionalen Wert, der sich auf die nach heutigen Erkenntnissen unbekannten Nutzwerte bekannter Arten sowie mögliche Nutzwerte noch nicht bekannter Arten bezieht. Angesichts der Tatsache, dass bislang nur ein Bruchteil selbst der weltweit bekannten Pflanzenarten hinsichtlich in ihnen enthaltener Wirkstoffe untersucht sind, kommt dem Offenhalten einer zukünftigen Verfügbarkeit biologischer Ressourcen erhebliche Bedeutung zu. Drittens dem indirekten Wert für die Aufrechterhaltung ökosystemarer Prozesse, wie etwa des Wasserund Kohlenstoffkreislaufs oder der Absorption und Umwandlung von Schadstoffen. Zur Frage, welche Bedeutung die Organismenvielfalt für die Erhaltung der Leistungsfähigkeit der Biosphäre hat, bestehen allerdings nach wie vor erhebliche Kontroversen und Forschungsbedarf. Neben dem sich aus den drei Komponenten zusammensetzenden, nutzungsabhängigen ,ökonomischen Gesamtwert" ist auch ein nutzungsunabhängiger Existenzwert zu berücksichtigen, der sich aus dem bloßen Wissen um die Existenz bestimmter Arten ergibt.

Insgesamt lässt sich aus all dem die Forderung ableiten, eine möglichst umfangreiche Biodiversität auf der gesamten Fläche, d. h. nicht nur auf biodiversitätsreichen bzw. -wertvollen Flächen, für die Zukunft zu erhalten bzw., falls notwendig, zu erhöhen.

Wechselwirkungen dieser ressourcenbezogenen Regel bestehen - in unterschiedlicher
Form und Intensität - mit den meisten der anderen Regeln, z. B.:

- Nachhaltige Nutzung der Umwelt als Senke. Die Verletzung der Umwelt durch Emissionen von Treibhausgasen, Schadstoffen oder Anreicherungen von Nährstoffen in Böden und Gewässern stellt eine Bedrohung für die erneuerbare Ressource Biodiversität dar. Hier ergeben sich Verknüpfungen z. B. zur klimabezogenen Adaptationsforschung, etwa im Bereich der Land- und Forstwirtschaft.

- Gewährleistung der Grundversorgung. Relevante Themenfelder sind hier insbesondere die Ernährungssicherheit, die Agrobiodiversität oder die Gesundheitsversorgung mit Hilfe von Wirkstoffen aus genetischen bzw. biologischen Ressourcen.

- Selbständige Existenzsicherung. Diese Regel ist in dem Maße relevant, wie Einkunftsquellen von Menschen von der hinreichenden Existenz bestimmter Pflanzenoder Tierarten abhängen.

- Gerechte Verteilung der Umweltnutzungsmöglichkeiten. Diese Leitlinie ist z. B. von Belang, wenn für bestimmte Länder ein fairer Zugang $\mathrm{zu}$ biologischen bzw. genetischen Ressourcen durch Biopiraterie untergraben wird oder wenn angemessene Regelungen zum Vorteilsausgleich in solchen Situationen getroffen werden sollen.

- Erhaltung des Human- und Wissenskapitals. Angesichts der bestehenden erheblichen Wissens- und Umsetzungsdefizite kommt der Erzeugung, Erhaltung und angemessenen Verwendung von Wissen in diesem Bereich große Bedeutung zu. Hierbei spielt das so genannte indigene Wissen der jeweils einheimischen Bevölkerung eine zunehmend wichtige Rolle.

- Erhaltung der kulturellen Funktion der Natur. Bedeutsam ist in diesem Zusammenhang, dass sich „Landschaften von charakteristischer Eigenart und Schönheit" (die diese kulturelle Funktion meist in besonderer Weise erfüllen) in der Regel durch einen hohen oder „wertvollen“ Biodiversitätsreichtum auszeichnen.

- Partizipation an gesellschaftlichen Entscheidungsprozessen. Angesprochen sind hier insbesondere Entscheidungen über Maßnahmen zum Schutz der Biodiversität. 
- Internalisierung externer ökologischer und sozialer Kosten. Diese Regel stellt etwa mit Blick auf die Phänomene Flächenverbrauch, Versiegelung und Landschaftszerschneidung - bei denen typischerweise die Verursacher nicht für die Folgen ihres Tuns in Haftung genommen werden - eine besonders wichtige Komponente für die Lösung bzw. Entschärfung des Problems des Biodiversitätsrückgangs dar.

- Faire weltwirtschaftliche Rahmenbedingungen. Relevant ist hier beispielsweise die Frage des Eigentums an biologischen bzw. genetischen Ressourcen und dessen Regelung. Dabei führt die bisherige internationale Praxis etwa hinsichtlich der Patentierung von Lebendigem bzw. von ganzen Lebewesen $\mathrm{zu}$ teilweise erheblichen Einschränkungen der Entwicklungsmöglichkeiten von ärmeren Staaten.

- Förderung der internationalen Zusammenarbeit. Im Vordergrund steht hier insbesondere das capacity building zu biodiversitätsbezogenen Fragen in Entwicklungsländern. Dies sollte sich insbesondere in der Unterstützung bedürftiger Staaten bei der Konzipierung einer nationalen Biodiversitätsstrategie, bei der Gestaltung und Finanzierung ihrer Umsetzung sowie bei Forschungsaktivitäten ausdrücken.

- Reflexivität und Resonanzfähigkeit der Gesellschaft. Hier wird zum einen der Blick auf die Frage gerichtet, inwieweit die Gesellschaft in der Lage ist, den Nutzen von Biodiversität zu erkennen und zu bewerten sowie auf Probleme in diesem Bereich angemessen $\mathrm{zu}$ reagieren. Zum anderen ist hier die Forderung enthalten, die Folgen von gesellschaftlichem bzw. politischem Handeln in Biodiversitätsfragen für die verschiedenen Akteure in die Überlegungen einzubeziehen.

Mit Hilfe der Nachhaltigkeitsregeln des HGFProjekts können also Hinweise zur Themenfindung und zu Zielen der Biodiversitätsforschung einerseits sowie zur Konzeption von Forschungsprozessen andererseits gegeben werden (z. B. Einbeziehung von lokalen Stakeholdern, capacity building in Entwicklungsländern usw.). Letzteres ist zum Teil bereits Praxis der vom BMBF geförderten Biodiversitätsforschung.

\section{Kontextuale Ebene}

Auf der kontextualen Ebene wurde im HGFProjekt auf Basis der Anwendung der Nachhaltigkeitsregeln und geeigneter Indikatoren der kontinuierliche Verlust von Biodiversität als eines von fünfzehn zentralen Nachhaltigkeitsproblemen Deutschlands identifiziert (Brandl, Kopfmüller, Sardemann 2003). Diese Einschätzung basiert nicht zuletzt auf dem Befund, dass die bereits 1998 von der damaligen Bundesumweltministerin Merkel geforderte Trendumkehr bei der Anzahl als gefährdet eingestufter Arten bislang nicht erreicht worden ist. Hierbei sind die Verursachungsbeiträge Deutschlands zum Biodiversitätsverlust jenseits der Grenzen Deutschlands (z. B. Gefährdung von Fischpopulationen durch Überfischung) oder in Entwicklungsländern (z. B. durch die Folgen der Rodung von besonders artenreichen Tropenwäldern usw.) noch nicht einbezogen.

Aufgrund des bislang - vor allem in den häufig besonders biodiversitätsreichen Entwicklungsländern - noch sehr begrenzten Wissens über den artenbezogenen Stand der Dinge besteht nach wie vor eine wesentliche Forschungsaufgabe in der Beantwortung der Frage, welche Arten in welchen Regionen in welchem Umfang vorhanden sind, welche Arten in welchem Umfang von wem für welche Zwecke genutzt werden und welche wesentlichen Eigenschaften diese Arten besitzen.

\section{Aktivitätsfelderebene}

Die vier im Projekt vertieft analysierten Aktivitätsfelder tragen wesentlich zum Biodiversitätsverlust bei (vgl. Jörissen, Heincke, Meyer 2003): Mobilität und Verkehr insbesondere über weiter steigenden Flächenverbrauch durch Verkehrswegebau, Infrastruktureinrichtungen und die dadurch entstehenden Zerschneidungseffekte; Wohnen und Bauen durch weiter steigenden Flächenverbrauch für Siedlungszwecke mit entsprechenden Folgen; Ernährung und Landwirtschaft in Form von industrieller Intensivlandwirtschaft auf einem großen Teil der Flächen, hohem Pestizid- und Herbizideinsatz und der Tendenz zur Konzentration auf die Nutzung weniger Pflanzen- und Tierarten; Freizeit und Tourismus durch Nutzung von Landschaften 
und Gegenden mit reicher Biodiversität als touristische Attraktion, die es aber gleichzeitig durch Übernutzung gefährdet (Küsten, Alpenregionen, Kulturlandschaften, nicht nur in Deutschland, sondern weltweit). Darüber hinaus tragen diese vier Aktivitätsfelder indirekt über hohe Treibhausgasemissionen, Emissionen von Luftschadstoffen oder den Eintrag von Nährstoffen (im Bereich Ernährung und Landwirtschaft) zu weiteren Gefährdungen der Biodiversität bei.

Hinzu kommt das im Projekt nicht bearbeitete Aktivitätsfeld Gesundheit, dessen Relevanz vergleichsweise weniger in seinem Beitrag zum Biodiversitätsverlust als darin besteht, dass hier in zunehmendem Maße biologische Ressourcen als Grundstoffe zur Bekämpfung von Krankheiten genutzt werden (könnten).

\section{Maßnahmenebene}

Für die Reduzierung bzw. den Stopp des Biodiversitätsrückgangs lassen sich insbesondere vier Ansatzpunkte unterscheiden: zunächst in einer übergeordneten Perspektive die Erarbeitung und Umsetzung einer nationalen Biodiversitätsstrategie mit möglichst quantifizierten Schutz- und Nutzungszielen (bemerkenswerterweise ist Deutschland einer der weniger Staaten, in denen eine solche Strategie bislang noch nicht existiert). Zweitens die angemessene Ausweisung und Vernetzung von Schutzgebietsflächen. Drittens die Reduzierung direkt oder indirekt biodiversitätsgefährdender Aktivitäten, etwa im Bereich der Landnutzung. Im HGF-Projekt wurden insbesondere Maßnahmen zur Reduzierung der Flächeninanspruchnahme und zum Schutz der Biodiversität, vor allem im agrarwirtschaftlichen Bereich, diskutiert. Zur Beschränkung des Flächenverbrauchswachstums werden dabei das bau- und planungsrechtliche Instrumentarium, Instrumente zum regionalen Flächenmanagement und marktwirtschaftliche Instrumente (Steuern, Abgaben, handelbare Flächenausweisungsrechte) betrachtet, in Bezug auf den Schutz der Biodiversität vor allem Maßnahmen zur Förderung biodiversitätsschonender landwirtschaftlicher Produktionsformen. Schließlich ist viertens die bessere finanzielle und personelle Ausstattung von Institutionen bedeutsam, um den Anforderungen an eine angemessene Erfassung, Dokumentation und Be- wertung der Biodiversität in bestimmten Regionen hinreichend gerecht werden zu können.

\section{Konsequenzen für die Forschungspolitik}

Die Analysen im HGF-Projekt bewegen sich auf einer mehr oder weniger hoch aggregierten Ebene. Der dort realisierte Mehrebenenansatz bestehend aus Zielformulierung in Form von normativen Nachhaltigkeitsregeln (globale Ebene), Diagnose von Nachhaltigkeitsproblemen (kontextuale Ebene), Problemverursachung (Aktivitätsfelderebene) und Maßnahmen (strategische Ebene) könnte jedoch als Vorbild für integrative Modellprojekte der Biodiversitätsforschung, z. B. auf lokaler Ebene, dienen. Ein solcher Forschungsansatz kann im Idealfall Wissen aller drei o. g. Wissenstypen produzieren und sinnvoll nur interdisziplinär (Sozialund Naturwissenschaften) und transdisziplinär (Einbeziehung von Praxis- oder Akteurswissen) realisiert werden.

Für die forschungspolitische Umsetzung empfiehlt sich folgendes Vorgehen:

- Zunächst die Ausschreibung einer deutlich begrenzten Anzahl von Modellprojekten der geschilderten Art.

- Als Themenfeld hierfür bietet sich die Biodiversität an aufgrund der vielfältigen sie beeinflussenden Faktoren und der unterschiedlichen Folgen ihrer Beeinträchtigung.

- Es sollte deutlich herausgestellt werden, dass es sich hierbei um „Projekte neuen Typs" in der oben beschrieben Form handelt. Dabei wird nach Wegen (inhaltlicher und organisatorischer Art) zu suchen sein, um die integrative Forschung mit der nach wie vor erforderlichen sektoralen Forschung (auch im Grundlagenbereich, etwa die Taxonomie betreffend) in angemessener Weise zu verknüpfen.

- Erfolgsaussichten bestehen nur, wenn die wichtigsten Projektbeteiligten bereits Erfahrung mit diesem Forschungstyp haben und diese auch nachweisen können. Dabei wird es besonders wichtig sein, hierfür angemessene Beurteilungskriterien zu finden, da natürlich „Erfahrung“ im engen Sinne in einem derartigen Neuland nur begrenzt möglich ist.

- Um die beabsichtigte Generierung neuer Wissenstypen und das capacity-building 
projektintern wie auch -extern mit Nachdruck zu verfolgen, sollte ein interdisziplinärer Beirat die Arbeit der Modellprojekte begleiten, bestehend aus WissenschaftlerInnen, die ebenfalls über entsprechende Erfahrungen verfügen. Ohne Zweifel stellt die Gewährleistung einer dem veränderten Forschungsansatz angemessenen Begutachtungs- und Evaluierungspraxis eine Herausforderung auch für die Forschungspolitik und -förderung dar, nicht zuletzt mit Blick auf die Suche nach hierfür geeigneten WissenschaftlerInnen.

- Die Modellprojekte sollten schließlich einer gründlichen Evaluierung unterzogen werden, um Erkenntnisse für die weitere Ausrichtung der integrativen Forschung zu gewinnen.

\section{Anmerkung}

1) Der nachfolgende Text stellt eine gekürzte Fassung dar des Thesenpapiers von Coenen, R.; Grunwald, A.; Kopfmüller, J., 2003: Nachhaltige Entwicklung und Globaler Wandel. Konsequenzen für die Forschung am Beispiel des Themenfelds Biodiversität. Karlsruhe

\section{Literatur}

Brandl, V.; Kopfmüller, J.; Sardemann, G., 2003: Die gegenwärtige Nachhaltigkeitssituation in Deutschland. In: Coenen, R.; Grunwald, A. (Hrsg.): Nachhaltigkeitsprobleme in Deutschland. Analyse und Lösungsstrategien. Berlin: edition sigma, S. 83-130

Grunwald, A., 2001: Integrative Forschung zum Globalen Wandel - Herausforderungen und Probleme. In: Coenen, R. (Hrsg.): Integrative Forschung zum Globalen Wandel - Herausforderungen und Probleme. Frankfurt a. Main, New York: Campus, S. 23-48

Hennen, L.; Krings, B.-J., 1998: Forschungs- und Technologiepolitik für eine nachhaltige Entwicklung. Arbeitsbericht Nr. 58 des Büros für TechnikfolgenAbschätzung beim Deutschen Bundestag. Bonn

Jörissen, J.; Heincke, M.; Meyer, B., 2003: Flächennutzung und Bodenschutz. In: Coenen, R.; Grunwald, A. (Hrsg.): Nachhaltigkeitsprobleme in Deutschland. Analyse und Lösungsstrategien. Berlin: edition sigma, S. 436-462

Kopfmüller, J.; Brandl, V.; Jörissen, J.; Paetau, M.; Banse, G.; Coenen, R.; Grunwald, A., 2001: Nachhaltige Entwicklung integrativ betrachtet. Konstitutive Elemente, Regeln, Indikatoren. Berlin: edition sigma
WBGU - Wissenschaftlicher Beirat der Bundesregierung Globale Umweltveränderungen, 2000: Welt im Wandel. Erhaltung und nachhaltige Nutzung der Biosphäre. Jahresgutachten 1999. Berlin u. a.: Springer

\section{Kontakt}

Jürgen Kopfmüller

Institut für Technikfolgenabschätzung und Systemanalyse (ITAS)

Forschungszentrum Karlsruhe $\mathrm{GmbH}$

Postfach 36 40, 76021 Karlsruhe

Tel: +49 (0) 7247 / 82 - 4570

Fax: +49 (0) 7247 / 82 - 4806

E-Mail: juergen.kopfmueller@itas.fzk.de

Internet: http://www.itas.fzk.de 